\title{
Methodological Problems in the Calculations on Amorphous Hydrogenated Silicon, a-Si:H
}

\author{
Alexander F. Sax and Thomas Krüger \\ Institut für Chemie, Karl-Franzens-Universität Graz, Strassoldogasse 10, A-8010 \\ Graz, Austria \\ alexander.sax@uni-graz.at, thomas.krueger@uni-graz.at
}

\begin{abstract}
Large silicon clusters (up to 230 atoms) with vacancies of different size were used to model the electronic structure of defects in amorphous silicon. We used a mechanical embedding technique, where a small core of atoms around a vacancy is surrounded by a larger number of bulk atoms. The electronic structure of the core was calculated with DFT methods, the bulk was treated with a semiempirical quantum chemical method. With this hybrid technique we investigated the structure of the cluster and ground and excited electronic states.
\end{abstract}

\section{Introduction}

Amorphous hydrogenated silicon, a-Si:H, is a cheap and technically versatile material used for example in solar cells. Its use is limited by the Staebler-Wronski effect [1], [2] which describes the degradation of a-Si:H manifesting in a considerable loss in photo-conductivity and dark conductivity after several hours of light exposure. This conductivity loss can, however, be fully reversed by thermal annealing in the dark. It is well known that a-Si:H has a large number of radical centers in the bulk due to unpaired electrons, called native dangling bonds. The existence of these dangling bonds in the bulk is one reason for the amorphous character of a-Si:H because not all silicon atoms are fourfold coordinated. Note, that missing covalent bonds lead immediately to geometry reorganization in the surrounding of the radical center and, thus, to deviations from the regular crystalline structure. The existence of dangling bonds in the amorphous material is also one explanation of the Staebler-Wronski effect, however, not the native dangling bonds are made responsible but so called light-induced or metastable dangling bonds. This explanation is based on the observation that after light exposure the number of dangling bonds, i.e. the spin density, increases by one to two orders of magnitude, after thermal annealing the spin density drops to the value typical for native dangling bonds. The metastable dangling bonds are produced from excitation of defect precursors.

Small angle X ray scattering investigations [3], [4], [5] show up to $5 \times 10^{19}$ microvoids per $\mathrm{cm}^{3}$ in a-Si:H. The microvoids are on average spherical with a mean radius of 3.3 to $4.3 \AA$, corresponding to 16 to 25 missing atoms, the distribution is, however, rather broad. The large internal surfaces are made by 
silicon atoms with dangling bonds which can form new bonds. As a result we expect a strong reorganization of the internal surface: The building of new bonds results in forces acting on old bonds. This leads to deviations from the standard bond length, bond angle and dihedral angles in crystalline silicon. It depends on the size and form of the void how strongly the reorganized structure deviates from the structure of crystalline silicon. One objective of our investigation was, therefore, to find out whether or not regularities in the structural reorganization exist.

Stretched bonds and deformed bond angles in the bulk as well as newly formed bonds influence the electronic spectrum of the system, so the defects in a-Si:H may be found at these microvoids. Moreover, it depends on the number of dangling bonds and the geometry of the void how many unpaired electrons couple to form new bonds and how many of them remain uncoupled. Such weakly coupled electrons can be easily excited and, thus, geometric arrangements leading to such electronic structures can be thought of being a defect precursor. Therefore, a detailed investigation of the electronic structure of a-Si:H was the second objective of this study.

\section{Methodological Problems}

The reliability of investigations using embedding techniques depends strongly on the correct size of the embedded core and the method chosen for its description as well as on the proper treatment of the surrounding bulk. In the core we must correctly describe the formation of new bonds or the reorganization of existing bonds which demands methods that account for electron correlation. Moreover, it is necessary to decide on how many silicon atoms define the core, only the atoms with dangling bonds, that is the atoms forming the internal surface of the void, or some more layers. Since the number of core atoms can become rather large density functional methods are certainly best suited for such investigations. The description of the bulk demands a method that allows for an elastic response of the bulk on forces that result from bond formation in the core. The bulk must only prevent the core atoms from collapsing into the void. The methods used for the bulk can, therefore, be at a much lower level than the method for the core. Whenever by the embedding the boundary between core and bulk region cuts through covalent bonds between core and bulk atoms, the proper treatment of this boundary region is crucial for the use of the embedding method.[6] Whenever the high and the low level methods describe the potential curves of the cut Si-Si bonds differently, i.e. the minima and the curvatures do not match, the convergence of the geometry optimization can be extremely slow or can completely fail and the final geometry parameters can be unreliable.

Molecular mechanics methods are frequently used as low level method but to fulfill the above mentioned requirement to match with the high level method re-parametrization of the force field parameters is often necessary. This is true for both, force fields used in solid state physics as well as force fields used in chemistry. Because the time for our project was limited we had to find a com- 
bination of existing methods that could be applied to our problem. We finally decided for a combination of the density functional BP86[7],[8] as the high level method and the semiempirical method AM1[9],[10] as the low level method. We used the ONIOM[11] embedding scheme which is implemented in the Gaussian98 software package. [12] The $6-31 \mathrm{G}^{*}$ basis set was used in all calculations. Justification for our choice of the density functional method was given in [13]. We used a semiempirical quantum chemical method instead of a force field because the Gaussian98 suite did not correctly handle the cluster with vacancies when the implemented force fields were combined with the density functional method, but this problem did not exist for semiempirical methods. We chose AM1 because with this method geometry optimization of large cluster converged, other semiempirical methods converged for small cluster but failed completely for the large ones.

The ability of the bulk to prevent a vacancy from collapsing into the void depends on its size. To find out how large the bulk must be we had to make test calculations on clusters of increasing size and of different form. For vacancies with one missing atom a cluster of 121 silicon atoms gave converged geometry data. For vacancies with more missing atoms cluster of more than 200 silicon atoms gave trustworthy results, sometimes even smaller clusters could be used.[14] Cluster size is the limiting factor in the applicability of this methodological approach because for a cluster with more than about 250 silicon atoms and vacancies in it the AM1 method shows serious convergence problems.

\section{Results}

\subsection{The structure of vacancies}

Whenever a single silicon atom is removed from a silicon crystal a simple vacancy (monovacancy) is created with four radical silicon atoms in tetrahedral arrangement. From the four atomic orbitals at the radical centers we can build four molecular orbitals, the one with the lowest orbital energy is totally symmetric and the remaining three orbitals form the basis for a threefold degenerate representation. Placing of four electrons in these four molecular orbitals results in a partial occupation of the degenerate orbitals yielding a degenerate electronic state which gives rise to a Jahn-Teller distortion of the nuclear frame. The local symmetry is lowered from $T_{d}$ to $C_{2}$ and the distances between two pairs of silicon atoms shrink from $3.82 \AA$ to about $2.45 \AA$.[14] We get, thus, two new Si-Si single bonds which are about $5 \%$ longer than the Si-Si single bonds in crystalline silicon. Because the symmetry is reduced to $C_{2}$ or $C_{s}$ the new bonds are not orthogonal to each other but twisted. This causes a deformation of the bond angles and dihedral angles in the surrounding of the vacancy. Due to JahnTeller distortion every vacancy contributes, thus, to a reduction of the crystalline character and to an increase of the amorphous character of the material.

Removing two adjacent atoms gives a vacancy with six radical centers in $D_{3 h}$ arrangement, two sets of three radical centers with local $C_{3}$ symmetry (equilateral triangle). For each set we get three molecular orbitals, one totally symmetric 
and one doubly degenerate. Putting three electrons in these three orbitals yields again a degenerate electronic state and, thus, gives rise to Jahn-Teller distortion. The local $C_{3}$ symmetry is lowered to $C_{2 v}$ and the radical centers can arrange in two possible structures: an acute angled and an obtuse angled isosceles triangle. We find in our calculations the obtuse angled structure with two newly formed Si-Si single bonds of about $2.5 \AA$. The bond angle between them is about $90^{\circ}$. Bonding interaction of the electrons in the two sets reduces the distances between the apex silicon atoms in the local triangles from $5.9 \AA$ in the crystal to 4.7 $\AA$. The Si-Si distances between the other silicon atoms shrink from $4.5 \AA$ in the crystal to $4.3 \AA$. The apex atoms in the obtuse angles triangles have three old $\mathrm{Si}-\mathrm{Si}$ single bonds to their neighbors and two new Si-Si single bonds to former radical centers, they are five-fold coordinated.[14] Again we find strong deviation from the crystalline structure in the neighborhood of such vacancies.

Vacancies with three[14] and four[15] missing silicon atoms show a much greater variety of structures. When three or four adjacent silicon forming a "linear" chain are removed the vacancy has the form of a tube. We find then always the formation of three or four new Si-Si single bonds which make the tube shrink. At both ends of the tube we find a single radical center. When four atoms forming a "circular" chain are removed a bond is formed that reminds at a twisted and stretched disilene, i.e. a twisted Si-Si double bond. When four silicon atoms that form a pyramid are removed we get the first vacancy which is rather hollow even after geometrical rearrangement.

These vacancies are clearly the limit of systems that can be treated with this methodological approach. Investigations of larger vacancies need certainly larger bulks and, therefore, methods that can handle them properly.

We calculated also systems with hydrogen placed in the vacancies. Preliminary results show clearly that formation of Si-H bonds leads to large shifts in the $\mathrm{Si}$-Si bonds, therefore, the geometries can show great structural differences compared with the vacancies without hydrogen. Hydrogen atoms that form strong $\mathrm{Si}-\mathrm{H}$ bonds at the internal surface of vacancies or microvoids could, thus, help to optimally separate the radical centers.

\subsection{The electronic structure of vacancies}

Light induced dangling bonds are thought to result from the electronic excitation of weak bonds.[14] So we calculated not only the geometric structure of the vacancy in its electronic ground state but also the lowest excited states. In a monovacancy the two newly formed Si-Si single bonds are so strong that the excitation energy is similar to that of a normal Si-Si bond in crystalline silicon. In vacancies with two or more missing atoms we obtain rather low lying excited singlet states which lie within the band gap of a-Si:H which is about $1.7 \mathrm{eV}$. These excited states result from the excitation of weak "bonds" like the weakly coupled electrons from the ends of the tubes mentioned above. The larger the distance between the radical center becomes the easier it is to excite an electron. Whether or not a low lying excited state can indeed be excited by light depends, however, on the oscillator strength of this excitation. Indeed, only few transitions to low 
lying excited states have a considerable oscillator strength so that the transition can lead to metastable dangling bonds. With our methods it is very laborious to find out whether or not such excited states have local minima. The existence of a local minimum is, however, a necessary condition for a stable defect structure.

Use of density functional methods does not pose big problems for most ground state investigations even when the ground state can be deferred from a large number of dangling bonds. The calculation of the excited states that have strong multiconfigurational character is, however, rather tricky. Unfortunately, calculation of excited states with density functional methods can not yet that routinely be done as the calculation of grounds states. Due to computer time and convergency reasons we have calculated the excitation spectra by conventional singly excited CI based on a Hartree-Fock wave function. This mixing of methods is, however, undesirable for a consistent investigation of a large series.

\section{Summary and Outlook}

With the described hybrid technique we are able to investigate structure and electronic spectrum of vacancies. We showed that formation of new bonds between dangling bonds yields a strong geometric reorganization of the cluster which is partially responsible for the amorphous character of the material. We could also show that such vacancies lead to electronic structures with low lying excited states. This technique has two main limits:

- The number of atoms in the cluster is limited to about 250 atoms, treatment of larger cluster is prevented by convergence problems caused by the semiempirical method as well as the drastically increasing computer time.

- Geometry optimization in excited states is not that routinely possible with the standard density functional methods as it is for ground states.

Therefore, our future work will focus on the selection and reparametrization of a force field for silicon that can be used in combination with density functional methods. Only then we have the preposition to enlarge the bulk and calculate the structure of larger vacancies or microvoids with 10 to 20 missing atoms.

Methodic developments in the density functional methods that allow a more efficient geometry optimization in excited electronic states would be highly welcomed.

\section{Acknowledgement}

This work was supported by grant No. S7910-CHE from the Austrian FWF (Fonds zur Förderung der wissenschaftlichen Forschung) within the scope of the joint German-Austrian silicon research focus (Siliciumschwerpunkt). 


\section{References}

1. Staebler, D. L., Wronski, C. R., Appl. Phys. Lett. 31 (1977) 292

2. Staebler, D. L., Wronski, C. R. J., Appl. Phys. 51 (1980) 3262

3. Williamson, D.L., Mahan, A. H., Nelson, B. P., Crandall, R. S., Appl. Phys. Lett. 55 (1989) 783

4. Mahan, A. H., Williamson, D.L., Nelson, B. P., Crandall, R. S., Phys. Rev. B 40 (1989) 12024

5. Remes, Z., Vanecek, M., Mahan, Crandall, R. S., Phys. Rev. B 56 (1997) R12710

6. Sauer, J., Sierka, M. J., Comput. Chem. 21 (2000) 1470

7. Becke, A. D., Phys. Rev. A 38 (1988) 3098

8. Perdew, J. P., Phys. Rev. B 33 (1986) 8822

9. Dewar, M. J. S., Zoebisch, E. G., Healy, E. F., J. Am. Chem. Soc. 107 (1985) 3902

10. Dewar, M. J. S., Reynolds, C. H., J. Comput. Chem. 2 (1986) 140

11. Dapprich, S., Komaromi, I., Byun, K. S., Morokuma, K., Frisch, M. J., J. Mol. Struct. (Theochem) 461-462 (1999) 1

12. M. J. Frisch, et al., Gaussian 98 (Revision A.7), Gaussian, Inc., Pittsburgh PA, 1998

13. Krüger, T., Sax, A. F., J. Comput. Chem. 22 (2001) 151

14. Krüger, T., Sax, A. F., Phys. Rev. B, 64 (2001) 5201

15. Krüger, T., Sax, A. F., Physica B, in press. 